\title{
Increased expression of plakoglobin is associated with upregulated MAPK and PI3K/AKT signalling pathways in early resectable pancreatic ductal adenocarcinoma
}

\author{
EKENE NWEKE ${ }^{1}$, MONDE NTWASA $^{2}$, MARTIN BRAND $^{3,4}$, JOHN DEVAR $^{1,5}$, \\ MARTIN SMITH $^{1,5}$ and GEOFFREY CANDY ${ }^{1}$ \\ ${ }^{1}$ Department of Surgery, Faculty of Health Sciences, University of Witwatersrand, Johannesburg, Gauteng 2193; \\ ${ }^{2}$ Department of Life and Consumer Sciences, University of South Africa, Johannesburg, Gauteng 1710; \\ ${ }^{3}$ School of Physiology, Faculty of Health Sciences, University of Witwatersrand, Johannesburg, Gauteng 2193; \\ ${ }^{4}$ Department of Surgery, Steve Biko Academic Hospital and The University of Pretoria, Pretoria, Gauteng 0002; \\ ${ }^{5}$ Department of Surgery, Chris Hani Baragwanath Hospital, Soweto, Johannesburg, Gauteng 1864, Republic of South Africa
}

Received July 17, 2019; Accepted January 29, 2020

DOI: $10.3892 / \mathrm{ol} .2020 .11473$

\begin{abstract}
Pancreatic ductal adenocarcinoma (PDAC) is one of the most lethal cancer types, and it is associated with a 5-year survival rate of $<10 \%$ due to limited early detection methods and ineffective therapeutic options. Thus, an improved understanding of the mechanisms involved in the early stages of PDAC tumorigenesis is crucial in order to identify potential novel diagnostic and therapeutic targets. The most common signalling aberrations in PDAC occur in the Wnt/Notch signalling pathway, as well as within the epidermal growth factor receptor (EGFR) pathway and its associated ligands, EGF and transforming growth factor- $\beta$. In addition, the RAS family of oncogenes, which act downstream of EGFR, are found mutated in most pancreatic cancer samples. Plakoglobin, a component of the EGFR signalling pathway, serves an important role in normal cell adhesion; however, its role in PDAC is largely unknown. The present study used transcriptome sequencing and focussed proteome microarrays to identify dysregulated genes and proteins in PDAC. The presence of upregulated plakoglobin expression levels was identified as a distinguishing feature between the PDAC microenvironment and normal pancreatic tissue. Furthermore, plakoglobin was demonstrated to be associated with the differential upregulation of the PI3K/AKT and MAPK signalling pathways in the tumour microenvironment, which suggested that it may serve an important role in PDAC tumourigenesis.
\end{abstract}

Correspondence to: Professor Monde Ntwasa, Department of Life and Consumer Sciences, University of South Africa, Calabash Building 211, 28 Pioneer Avenue, Florida Park, Cnr Christiaan de Wet and Pioneer Roads, Johannesburg, Gauteng 1710, Republic of South Africa E-mail: ntwasmm@unisa.ac.za

Key words: pancreatic ductal adenocarcinoma, $\gamma$-catenin, JUP, gene expression, P13K/AKT, MAPK signalling

\section{Introduction}

Despite the overall reduction in the number of cancer-related deaths following improved therapeutic options, the number of pancreatic ductal adenocarcinoma (PDAC)-related deaths continues to increase; it is associated with a median overall survival of $<1$ year and $<10 \%$ survival at 5 years (1). Thus, an improved understanding of the mechanisms mediating PDAC tumourigenesis is required.

Plakoglobin, also known as $\gamma$-catenin, is a member of the catenin family and is an important component of desmosomes and adherens junctions in mammalian cells, where it facilitates cell-cell adhesion by linking desmosomal cadherins to the cytoskeleton $(2,3)$. Plakoglobin can also be found localised in the nucleus, where it exerts nuclear functions, such as inhibiting the Wnt $/ \beta$-catenin signalling pathway through regulating the transcription of specific components within the pathway $(4,5)$. Nonetheless, the role that plakoglobin serves in tumorigenesis is disputed; whilst the majority of previous studies reported that plakoglobin suppressed tumour growth, others observed that plakoglobin exhibited pro-oncogenic features (5-9). For example, the overexpression of plakoglobin in SV40-transformed 3T3 cells suppressed tumour formation upon transplantation into syngeneic mice, and upon co-transfection of plakoglobin with $\mathrm{N}$-cadherin, this tumour suppressive effect was considerably more effective than plakoglobin overexpression alone. Similarly, the overexpression of plakoglobin in a human renal carcinoma cell line that lacked the expression of cadherins or $\beta$-catenin also suppressed tumour formation in nude mice (6). In addition, plakoglobin reportedly affected several downstream signalling molecules involved in tumour suppressive pathways; plakoglobin reportedly regulated the expression and stability of the metastasis suppressor protein, $\mathrm{Nm} 23$, as well as the

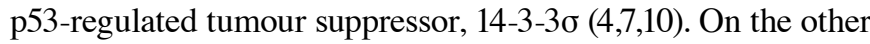
hand, a recent study demonstrated that high expression levels of plakoglobin promoted metastasis in invasive micropapillary carcinoma of the breast by promoting tumour cluster formation through activating PI3K/AKT/Bcl-2 signalling (9). 
As a closely related homologue of $\beta$-catenin, a crucial transcriptional regulator of the canonical Wnt signalling pathway, plakoglobin shares structural and functional similarities with $\beta$-catenin (11). It has previously been demonstrated that plakoglobin competes with $\beta$-catenin, which leads to aberrations in Wnt signalling; both $\beta$-catenin and plakoglobin were observed to bind to the transcriptional co-activator, transcription factor 7-like 2 (also known as Tcf4), although the latter bound with stronger affinity, and their co-expression led to the overall net inhibition of Wnt signalling $(12,13)$. Plakoglobin was also discovered to activate T-cell factor/lymphocyte enhancer factor (TCF/LEF) transcriptional activity in the absence of $\beta$-catenin (11), and the decrease in plakoglobin expression levels correlated with poorer prognosis in malignancies such as non-small lung cancer (14).

PDAC originates from both acinar and ductal cells and the well-described mechanism of tumorigenesis involves the early genetic alteration in the oncogene, $K-R A S$, whereby the expression of $K-R A S^{G l 2 D}$ is thought to induce the disease (15-17). However, studies in mice models have demonstrated that the expression of $K R A S^{G 12 D}$ in mature pancreatic acinar cells was insufficient to induce the PDAC precursor, pancreatic intraepithelial neoplasia (18), and that the accumulation of mutations in P16, TP53 and SMAD4 (15-17) are also required for the cancer to progress. Thus, nodal regulators of cellular responses have been proposed as potential strategies for inhibiting PDAC formation (18). It was noted in a previous study that compared with pancreatic acinar cells, ductal cell developed more rapidly into PDAC in the presence of $K-R A S$ and TP53 mutations (19). Thus, the discovery of other mechanisms dependent or independent of $K-R A S$ is required to understand the induction, development and progression of early-stage PDAC. Genetic analysis of 24 patients with advanced PDAC revealed that twelve core signalling pathways were dysregulated in 67\% of PDAC tumours, including Hedgehog, Wnt/Notch, K-RAS, small GTPase, transforming growth factor (TGF)- $\beta$ and integrin signalling. Notably, despite the heterogeneity of these altered genes amongst the patients, all PDAC tumours demonstrated alterations in the Wnt/Notch and Hedgehog signalling pathways (20). Further investigations have observed the induction of several mitogenic signalling pathways by various growth factors in PDAC (21-24). Altogether, these studies suggested that alterations in single molecules in PDAC present little opportunity for drug development, but targeting downstream effectors at nodal points, which control biological processes such as metabolism, cell migration and apoptosis, may be more feasible interventions for drug development. In the present study, the expression of dysregulated genes in early PDAC tumours, as well as differentially regulated signalling pathways in the PDAC tumour microenvironment, were investigated compared with normal pancreatic tissue.

\section{Materials and methods}

Patient studies. The present study was approved by The Human Research Ethics Committee of The University of Witwatersrand (approval no. M150778; Johannesburg, South Africa). Informed, voluntary consent was obtained from all patients. Samples were obtained from consented patients (age range 52-67 years) at Chris Hani Baragwanath Hospital, Johannesburg South Africa from January 2014 to June 2016. PDAC tumour samples and paired non-malignant pancreatic tissue samples ( $\geq 2 \mathrm{~cm}$ away from the tumour) were obtained from nine patients who underwent a pancreaticoduodenectomy for clinically resectable, early stage PDAC (Table I). It is important to note that patients recruited for this study were representative of different clinical stages, thus observations made within the study were attributed to PDAC in general. Biopsies were stored in $1 \mathrm{ml} \mathrm{RNAlater}$ RNA stabilization reagent (cat. no. 76106; Qiagen, Inc.) and subsequently homogenized in $600 \mu \mathrm{l}$ AllPrep DNA/RNA Mini kit lysis buffer (cat. no. 80204; Qiagen, Inc.) using a TissueRuptor (cat. no. 9001272; Qiagen, Inc.).

RNA extraction. Total RNA was extracted from PDAC tumour tissue and non-malignant pancreatic tissue using an AllPrep DNA/RNA Mini kit (cat. no. 80201; Qiagen, Inc.) and DNase digestion was performed using a DNase kit (cat. no. AMPD1; Sigma-Aldrich; Merck KGaA), according to the manufacturers' protocols. Total RNA was quantified using a NanoDrop ND-1000 UV spectrophotometer and a ratio of absorbance at 260 and $280 \mathrm{~nm}$ of $>1.8$ was observed in all samples.

Gene expression profiling using RNA sequencing. Total RNA extracted from tissue samples obtained from patients 7 and 8 (Table I) were treated with DNase to remove genomic DNA. The NEB Next rRNA Depletion kit (human/mouse/rat) (New England Biolabs Inc.; cat no. E6310S) was subsequently used to remove the ribosomal RNA from the samples. The mRNA was converted into cDNA using the Ultra RNA Library Prep kit (New England Biolabs Inc.; cat no. 7530L) and subsequently sequenced using the Illumina MiSeq system. The CLC Genomics Workbench version 11 software (www. qiagenbioinformatics.com/products/clc-genomics-workbench) was used for data normalization and differential gene analysis, with HG19 serving as the reference genome.

Biomarker filtering analysis. The biomarker filtering analysis tool available in the Ingenuity pathway analysis (IPA) software v01-08 (https://www.qiagenbioinformatics. com/products/ingenuity-pathway-analysis/ was used to identify novel potential biomarkers. Microsoft Excel files containing the differentially expressed genes (DEGs) obtained from RNA sequencing of tissue samples were uploaded onto the IPA platform and the biomarker filtering tool facilitated the identification of relevant and novel biomarkers based on the applied filters. The following filters were used: Species (Human); Tissues and cell lines (Pancreatic cancer cell lines); Node types (All nodes except complexes and groups): Diseases (Cancer); Biofluids (Blood); Molecules (All types); Biomarker (Not a known biomarker). Manual filtering was also conducted, including searching the NCBI database (https://www.ncbi.nlm.nih.gov/pubmed/) and pancreatic cancer database (http://pancreaticcancerdatabase. org/index.php) to further refine the biomarker analysis.

Validation by reverse transcription-quantitative $P C R$ $(R T-q P C R)$. The mRNA expression levels of potential biomarkers were validated using $\mathrm{RT}^{2}$-qPCR primer assays (Qiagen, Inc.), according to the manufacturer's protocol. The cycling condition was set as follows: $95^{\circ} \mathrm{C}$ for $10 \mathrm{~min}(1$ cycle), $95^{\circ} \mathrm{C}$ for $15 \operatorname{secs}\left(45\right.$ cycles) and $60^{\circ} \mathrm{C}$ for $1 \mathrm{~min}$ ( 45 cycles). At 
Table I. Characteristics of patients enrolled in the present study.

\begin{tabular}{lllllllll}
\hline No. & Sex & Age & Alcohol use & Diabetes & Differentiation & TNM & Stage & Alive 12/12 \\
\hline 1 & Male & 67.1 & No & Yes & Mod diff & T3N1M0 & IIB & Yes \\
2 & Male & 55.8 & Yes & No & Well diff & T1N0M0 & IA & Yes \\
3 & Female & 62.7 & No & Yes & Well diff & T2N1M0 & IIB & Yes \\
4 & Male & 63.5 & Yes & No & Mod diff & T3N1M0 & IIB & Yes \\
5 & Male & 54.3 & Yes & No & Poor diff & T2N1M0 & IIB & Yes \\
6 & Male & 52.2 & Yes & Yes & Well diff & T3N1M0 & IIB & Yes \\
7 & Female & 57.3 & No & No & Mod diff & T3N1M0 & IIB & Yes \\
8 & Male $^{\text {a,b }}$ & 43.4 & No & No & Mod diff & T3N1M0 & IIB & Yes \\
9 & Female $^{\mathrm{b}}$ & 52.6 & No & No & Mod diff & T2N1M0 & IIA & Yes \\
\hline
\end{tabular}

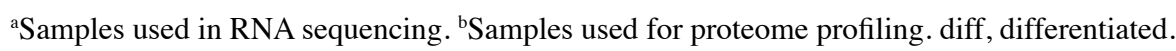

the end of the cycles the $\mathrm{Cp}$ values were obtained to be used for analysis. Kits were purchased targeting JUP (cat on pancreatic tissue. no. PPH07138F), COPG (cat. no. PPH07138F), TCIRG1 (cat. no. PPH09294A) and the reference gene MRPL19 (cat. no. PPH09294A), a housekeeping gene whose expression remains unchanged in PDAC carcinogenesis (25). A Roche LightCycler-480 was used with the cut off $\mathrm{Cp}$ set at $3 \mathrm{~s}$, according to the manufacturer's protocol. Experiments adhered to the Minimum Information for Publication of Quantitative Real-Time PCR Experiments guidelines, including performing three independent experiments (26). Expression levels were quantified by uploading the data into the Relative Expression Software Tool (REST) software (27).

Protein extraction and quantification. Total protein from $20 \mathrm{mg}$ PDAC and non-cancerous pancreatic tissue was extracted using a lysis buffer [ $(0.5 \%$ igepal, $0.5 \%$ sodium deoxycholate, $0.1 \%$ sodium dodecyl sulphate, $150 \mathrm{mM}$ sodium chloride and $50 \mathrm{mM}$ Tris- $\mathrm{HCl}(\mathrm{pH} 7.5)$ containing a protease inhibitor cocktail of aprotinin $(0.5 \mu \mathrm{g} / \mathrm{ml})$ and PMSF $(1 \mathrm{mM})]$. Tissues were homogenized using TissueRuptor and centrifuged at 2,000 x g for $10 \mathrm{~min}$ at $4^{\circ} \mathrm{C}$. The supernatant was transferred into a new tube and the total protein was quantified using the Quick Start ${ }^{\mathrm{TM}}$ Bradford protein assay kit (cat. no. 5000006; Bio-Rad Laboratories, Inc.), according to the manufacturer's protocol.

Focused proteomic profiling on pancreatic tissue using Human Oncology Proteome Profiler Array. Protein expression profiling was conducted to determine the relative expression levels of 84 cancer-related proteins using the Proteome Profiler Human XL Oncology Array kit (cat. no. ARY026; R\&D Systems Europe, Ltd.), according to the manufacturer's protocol. Quick Spots image analysis software vPCM.22.0.0.i (R\&D Systems Europe, Ltd.) was used to measure the intensity of each spot and identify differentially expressed proteins (DEPs). The intensity of each protein was compared between the tumour and corresponding normal pancreatic tissues, and differentially expressed proteins (DEPs) with $\mathrm{P}<0.05$ were selected. Briefly, the membranes were incubated at room temperature in $2 \mathrm{ml}$ block buffer and placed on a rocking platform for $1 \mathrm{~h}$. Subsequently, $500 \mu \mathrm{l}$ Array Buffer 4 was added and the membranes were incubated overnight at $4^{\circ} \mathrm{C}$. Following incubation, the membranes were washed three times with $1 \mathrm{ml}$ wash buffer. Then, $30 \mathrm{ml}$ detection antibody cocktail was added to $1.5 \mathrm{ml} 1 \mathrm{X}$ Array buffer $4 / 6$ and incubated at room temperature with the membrane on a rocking platform shaker for $1 \mathrm{~h}$. Subsequently, $2 \mathrm{ml}$ diluted horseradish peroxidase conjugated-streptavidin was added to the membrane and incubated at room temperature for $30 \mathrm{~min}$. Finally, the membrane was washed as previously described and $1 \mathrm{ml}$ Chemi reagent mix was added to the membrane. Membranes were visualised using a Bio-Rad ChemiDoc imaging system (Bio-Rad Laboratories, Inc.).

Statistical and bioinformatics analysis. Statistical analysis on three biological replicates was performed using Microsoft Office Excel (2016) and data are presented as the mean \pm SEM. Differences between groups were analysed using Student's t-test. $\mathrm{P}<0.05$ was considered to indicate a statistically significant difference. The Reactome database (28) and PANTHER version 6 software (29) were used to identify dysregulated pathways $(\mathrm{P}<0.05)$. For statistical analysis of the real-time PCR data, the Pair Wise Fixed Reallocation Randomisation Test was performed by the REST software.

\section{Results}

JUP is upregulated in early resectable PDAC tumours. Differential gene expression analysis of the sequenced transcriptomes from patients 1 and 2 revealed that 4,911 genes were commonly upregulated and 5 were downregulated (Fig. 1A). Using the IPA biomarker module and aforementioned filter parameters, JUP encoding plakoglobin, $C O P G$ encoding coatomer protein complex, subunit gamma and TCIRG1 encoding T-cell immune regulator 1 were identified as potential biomarkers. Validation of these genes using RT-qPCR demonstrated that JUP expression levels were increased in all PDAC samples compared with the corresponding normal pancreatic tissue $[\mathrm{p}(\mathrm{H} 1)=0.012]$ (Fig. 1B). However, the expression levels of $C O P G$ in PDAC tissues were not significantly different compared with normal tissues, with a probability of the alternate hypothesis $[\mathrm{p}(\mathrm{H} 1)=0.703]$. TCIRG 1 expression levels were also unchanged between normal and PDAC tissue samples $[\mathrm{p}(\mathrm{H} 1)=0.597]$. Although the expression levels of JUP were significantly increased in tumour tissues, it was observed to have a wide $95 \%$ confidence interval of $1.25-1,856.39$. 
Table II. Top upregulated pathways identified in tumours using the Reactome and PANTHER databases following RNA sequencing.

Number of

Pathways

P-value

genes involved

Insulin/IGF pathway-mitogen activated protein

$2.19 \times 10^{-2}$

19

kinase/MAP kinase cascade

Integrin signaling pathway

$9.65 \times 10^{-15}$

Ras pathway

$1.10 \times 10^{-3}$

39

PDGF signaling pathway

$1.06 \times 10^{-6}$

74

EGF receptor signaling pathway

$7.72 \times 10^{-6}$

70

Angiogenesis

$5.58 \times 10^{-7}$

88

CCKR signaling map

$1.16 \times 10^{-6}$

VEGF signaling pathway

$4.11 \times 10^{-2}$

$B$ cell activation

$4.11 \times 10_{-2}$

Notch signaling pathway

$2.6 \times 10^{-6}$

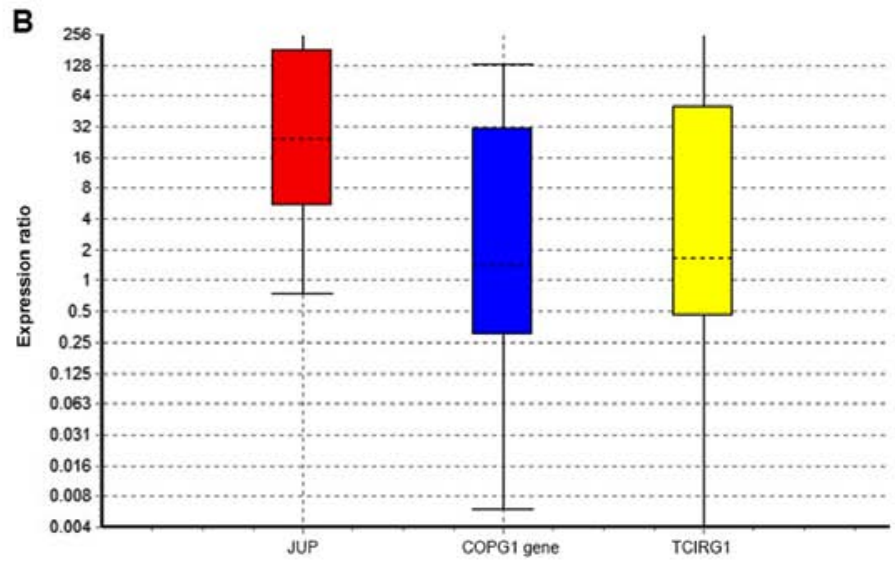

Figure 1. Gene expression profiles in PDAC tumours. (A) RNA sequencing was performed, and Venn diagrams are used to present upregulated and downregulated genes in early stage PDAC patients (patients 7 and 8). A total of 4,911 genes were found to be upregulated, whereas only 5 genes were observed to be downregulated. (B) Reverse transcription-quantitative PCR was performed to assess the expression levels of $J U P, C O P G$ and $T C I R G 1$ in PDAC tumours (n=9). The Boxplot was generated using REST software and the boxes represent the interquartile range while dotted lines represent the median gene expression. PDAC, pancreatic ductal adenocarcinoma; N1, normal tissue for patient 7; T1, PDAC tumour tissue for patient 7; N2, normal tissue for patient 8; T2, PDAC tumour tissue for patient 8 .

Dysregulated pathways in PDAC identified by gene expression analysis. Bioinformatics analysis predicated that the insulin growth factor-1 (IGF-1) pathway and mitogen-activated protein kinase (MAPK) kinase signalling cascade were the most upregulated pathway in PDAC tumours (Tables II and SI). The other most upregulated and active signalling pathways were the integrin $\left(\mathrm{P}=9.65 \times 10^{-15}\right)$, RAS $\left(\mathrm{P}=1.10 \times 10^{-3}\right)$, platelet-derived growth factor $\left(\mathrm{P}=1.06 \times 10^{-6}\right)$, epidermal growth factor receptor (EGF; $\left.\mathrm{P}=7.72 \times 10^{-6}\right)$, angiogenesis $\left(\mathrm{P}=5.58 \times 10^{-7}\right)$, cholecystokinin receptor $\left(\mathrm{P}=1.16 \times 10^{-6}\right)$, Vascular endothelial growth factor $\left(\mathrm{P}=4.11 \times 10^{-2}\right)$, B-cell activation $\left(\mathrm{P}=4.11 \times 10^{-2}\right)$ and $\operatorname{Notch}\left(\mathrm{P}=2.6 \times 10^{-6}\right)$ signalling pathways (Tables II and SII-SX). Pathways found to be downregulated in PDAC included the proton/oligopeptide co-transportation system, Golgi-ER trafficking and MHC II antigen presentation pathways (Table III). Additional analysis using Reactome confirmed the dysregulation of these pathways.
Upregulated proteins and their signalling pathways. Protein expression profiling of PDAC and normal tissue identified nineteen significantly upregulated proteins and no downregulated proteins (Fig. 2A and Table SXI). The adherens junction protein, nectin- 4 , was observed to be the most significantly overexpressed $\left(\mathrm{P}=4.85 \times 10^{-6}\right)$, followed by $\mathrm{p} 27^{\mathrm{Kip} 1}\left(\mathrm{P}=5.966 \times 10^{-3}\right)$, mucin-1 (MUC-1; $\left.\mathrm{P}=8.420 \times 10^{-3}\right)$ and gelsolin-like actin-capping protein $\left(\mathrm{CapG} ; \mathrm{P}=1.021 \times 10^{-2}\right.$; Fig. 2B). Further analysis of these proteins demonstrated the enrichment of several pathways (Fig. S1 and Table IV).

\section{Discussion}

In the present study, using data produced by transcriptome sequencing and a focused proteome microarray, plakoglobin was found to be significantly upregulated in early stage PDAC tumours compared with normal pancreatic tissue. In addition, analysis of dysregulated pathways within the 
Table III. Top downregulated pathways identified in tumours using the Reactome and PANTHER databases following RNA sequencing.

\begin{tabular}{llc}
\hline Pathways & \multicolumn{1}{c}{ Genes involved } & P-value \\
\hline $\begin{array}{l}\text { Proton/oligopeptide co-transportation } \\
\text { (Drug transportation) }\end{array}$ & SLC15A1, SLC15A2, SLC15A4 & $1.21 \times 10^{-7}$ \\
Organelle trafficking & KIFC, GALNT1, KIF5A, MANIA1 & $1.12 \times 10^{-3}$ \\
Membrane trafficking & SEC23B, KIF5C, GALNT1, KIF5A, DVL2, MANIA1 & $2.82 \times 10^{-3}$ \\
MHC Class II antigen presentation & SEC23B, KIF5C, KIF5A & $3.87 \times 10^{-3}$ \\
(Immune activation) & &
\end{tabular}

A

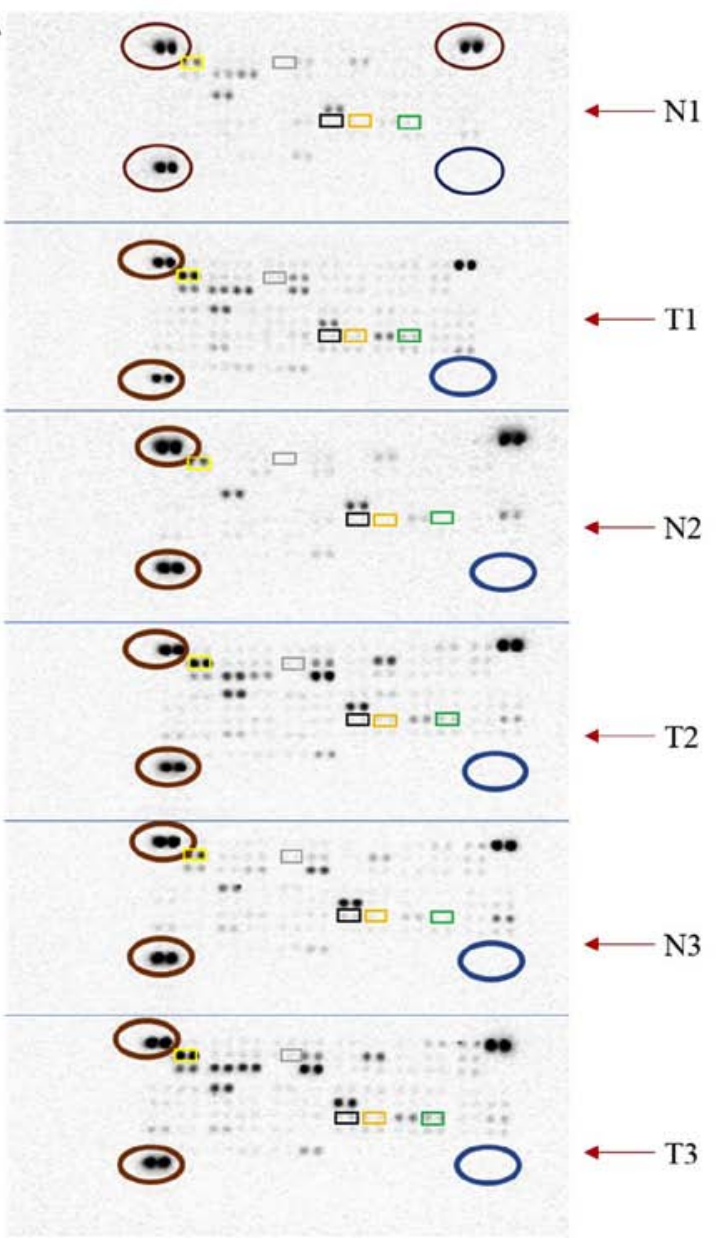

\section{B}

a Statistically significant upregulated proteins

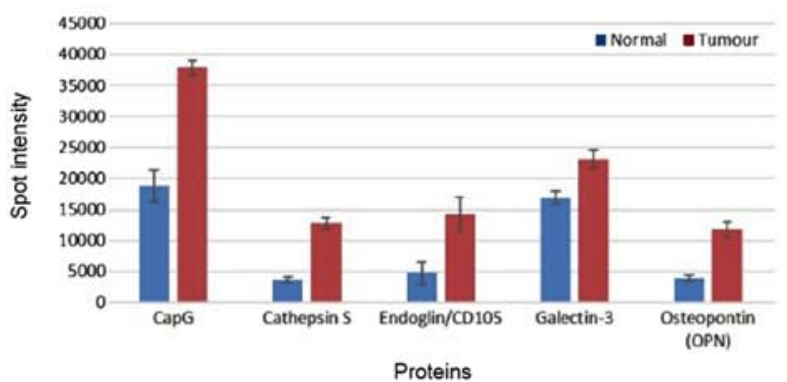

b Statistically significant upregulated proteins

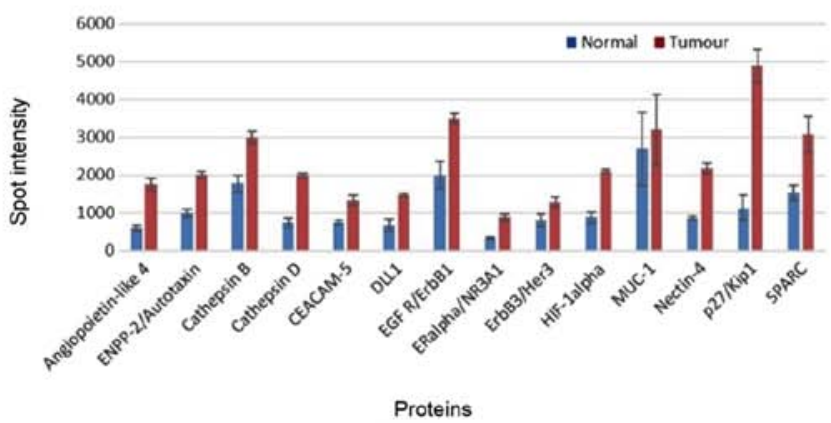

Figure 2. Differential protein expression levels using Human Oncology Proteome Profiler arrays. (A) Arrays demonstrating the protein expression profile for PDAC tumour tissue and normal tissue from 3 patients (patients 7,8 and 9). Each paired spot represents a protein and protein expression increases as spot intensity increases. Paired spots at the top left, bottom left and top right (all presented as red circles across the arrays) served as positive controls, whilst paired spots at the bottom right (shown in blue circles across all arrays) served as negative controls. Top 5 significantly overexpressed proteins are indicated in rectangles: Nectin (orange), p27 (green), MUC-1 (black), CapG (yellow) and Cathepsin D (grey). (B) Bar charts of upregulated proteins. Proteins in the top chart have a maximum spot intensity of 40,000, while those in the bottom chart have a maximum spot intensity of $\sim 6,000$. The blue bar indicates normal expression and red indicates tumour tissue expression. N1, normal tissue for patient 7; T1, tumour for patient 7; N2, normal tissue for patient 8; T1, tumour tissue for patient 8; N3, normal tissue for patient 9; T3, tumour for patient 9; MUC-1, mucin 1; CapG, gelsolin-like actin-capping protein.

PDAC tissue revealed that the IGF-1 and the EGF receptors (IGF1R and EGFR, respectively), as well as components of the K-RAS signalling pathway were also upregulated. Plakoglobin, also known as $\gamma$-catenin, is encoded by the junction plakoglobin (JUP) gene and belongs to the catenin protein family (8). It serves a critical role in cell adhesion as an important component of desmosomes and adherens junctions; through interacting as a complex with $\alpha$ - or $\beta$-catenin, plakoglobin associates with the cytoplasmic domains of E-cadherin or N-cadherin to facilitate the linkage between adherens junctions and the cytoskeleton $(30,31)$. Recently, plakoglobin was identified as a transcriptional factor involved in the modulation of WNT signalling (8). It was also demonstrated to act as a global regulator of gene expression; it was found to interact 
Table IV. Top upregulated pathways identified in tumours from proteome profiler arrays. Generated using Reactome.

\begin{tabular}{lll}
\hline Upregulated pathway & P-value & \multicolumn{1}{c}{ Candidate proteins } \\
\hline ErbB signaling pathway & $4.9 \times 10^{-5}$ & EGFR, ERBB3, CDKN1B \\
MHC class II antigen presentation & $5.74 \times 10^{-5}$ & CTSS, CTSB, CTSD \\
Immune system & $8.14 \times 10^{-5}$ & CTSD, CDKN1B, ERBB3, CTSB, CTSS, EGFR \\
Signalling pathway in endosomal TLR & $1.68 \times 10^{-4}$ & EGFR, CDKN1B, ERBB3 \\
P13/AKT signalling in cancer & $1.8 \times 10^{-4}$ & ERBB3, EGFR, CDKN1B \\
\hline
\end{tabular}

with p53 transcription regulatory elements to transcriptionally modulate the TCF/LEF family in an APC-dependent manner $(11,32)$, and upon addition of exogenous plakoglobin to a mesothelioma cell line, plakoglobin accumulated in the nucleus and exhibited transcriptional activity (11). Overall, this suggests that plakoglobin may serve as a transcription factor.

The insulin/IGF-1R, which may signal through the rat sarcoma (RAS) signalling pathway, is important for transducing signals that promote the development of pancreatic cancer; the crosstalk between insulin/IGF and G protein-coupled receptor signaling was observed to depend on mTOR complex 1 in pancreatic cell lines (33). The findings from the present study suggested that the MAPK and PI3K/AKT signalling pathways may be upregulated in the early PDAC tumour microenvironment; however, the mechanism behind this dual signaling remains largely unknown. Alterations in single or groups of genes involved in one pathway often trigger tumorigenic cellular behaviour. It has been reported that $\sim 95 \%$ of PDAC tumours harbour activating $K-R A S$ mutations (34) and RAS is a common signaling component of both the MAPK and PI3K/AKT signaling pathways; however, although RAS isoforms are structurally similar, they demonstrate distinct roles in different subcellular environments to mediate different signalling pathways (35). A comprehensive analysis of active signaling pathways in pancreatic cancer demonstrated that 12 of these pathways were often associated with single or multiple gene alterations that arose from different signaling pathways (20). Notably, in the present study, $N-R A S$ was the only isoform identified as being upregulated in PDAC through transcriptome sequencing (Table SIII); $N$-RAS mutations are frequently observed in melanoma (35).

Receptor tyrosine kinases such as the IGF-IR and EGFR, which were both found to be upregulated in PDAC in the present study, transduce signals via the insulin receptor substrate-1 (IRS) to either the MAPK or PI3K/AKT signaling pathway to control cell proliferation and survival (33). EGFR expression levels are upregulated in $\sim 90 \%$ of pancreatic tumours, which contributes to chemoresistance (36-38). Thus, the combination of molecules found upregulated in the PDAC tumour microenvironment in the present study further suggested that the PI3K/AKT and MAPK signaling pathways may be upregulated in PDAC compared to within normal pancreatic tissue.

The precise signalling role plakoglobin serves during pancreatic carcinogenesis is largely unclear; however, one study reported that plakoglobin promoted the phosphorylation of AKT and ERK in an EGFR-dependent manner, and that its overexpression promoted cell mobility and migration (39). The data from the present study demonstrated that both EGFR and insulin/IGF-1R were upregulated in the PDAC tumour microenvironment. In normal muscle tissue, plakoglobin bound to the insulin receptor and the p 85 regulatory subunit of PI3K to promote the activation of the PI3K-AKT-FoxO pathway and muscle growth; in this context, plakoglobin was tightly regulated by the E3 ligase, Trim-32; however, it is also important to note that changes in plakoglobin expression alone were insufficient to influence the signal transduction and muscle growth (40). In invasive micropapillary carcinoma of the breast, plakoglobin was observed to be overexpressed in metastatic tissue, and it contributed to metastasis through activating the PI3K/AKT/Bcl-2 signalling pathway (9). This suggested a critical role for plakoglobin in PI3K/AKT signalling. It has also been reported that both the MAPK and PI3K/AKT signalling pathways exist in pancreatic cancer cell lines and that the inhibition of both pathways leads to cell cycle arrest and apoptosis (41). In addition, plakoglobin was demonstrated to have an important role in maintaining cell adhesion in keratinocytes through inhibiting p 38 MAPK, hence, the loss of function of plakoglobin resulted in the activation of p38 MAPK in these cells (42). However, the activation of p38 is context-dependent; for example, insulin stimulated p38 in cultured mouse adipocyte cells but downregulated it in chick neuronal cells $(43,44)$. Overall, the findings from the present study, considered alongside the existing research, suggest that plakoglobin may be an important context-dependent factor in carcinogenesis. A conceptual schematic diagram to illustrate the signalling networks upregulated in early PDAC as well as the hypothesised mechanism of action exerted by plakoglobin is presented in Fig. 3.

Plakoglobin may also influence cellular outcomes through its nuclear functions. A candidate nuclear molecule identified in the present study was $M U C 1$, whose overexpression was reported in the proteomic study. A previous study observed that $\mathrm{MUCl}$ was transported in complex with plakoglobin to the nucleolus in HER2-positive primary invasive ductal breast carcinomas following treatment with heregulin/neuregulin-1 (HRG) (39). This HRG-induced nucleolar translocation of the plakoglobin-MUC1 complex was dependent on $M U C 1$, because a mutation in its cytoplasmic RRK motif prevented its nuclear transfer (45). Notably, both MUC1 and HER2 were upregulated in the proteomic study and the transcriptome sequence data demonstrated that HERl was also one of the genes representative of EGFR signalling (Table SV). In addition, both the transcriptomic and proteomic data analyses revealed further proteins directly or indirectly associated with the insulin/IGF-1 and EGFR signalling pathways (Fig. 2); this included the adherens junction protein nectin-4, which has previously been identified as a tumour-associated 


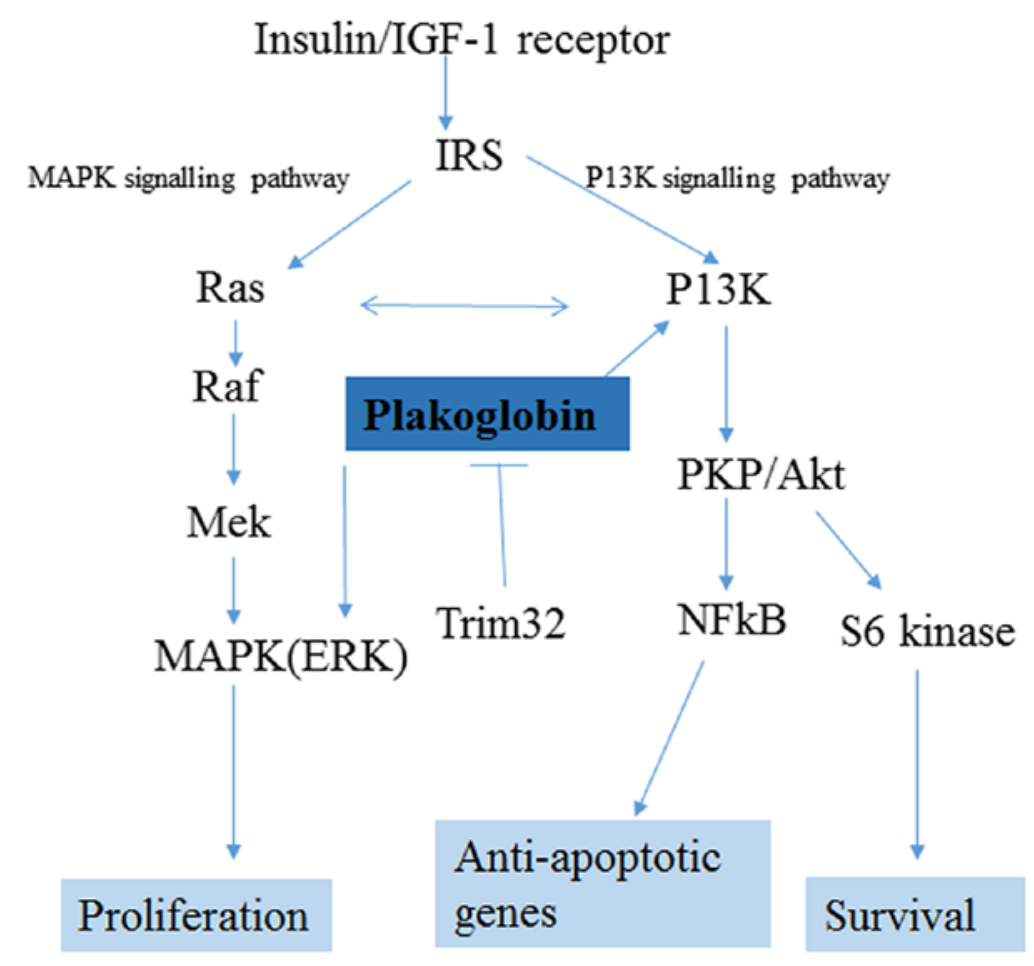

Figure 3. Proposed action of plakoglobin in pancreatic ductal adenocarcinoma. Receptor tyrosine kinases such as insulin/insulin-like growth factor-1 transduce signals through the insulin receptor substrate-1, which can either activate the MAPK or PI3K/AKT signalling pathway. There is evidence of crosstalk between RAS and PI3K in some contexts. Plakoglobin has been demonstrated to modulate PI3K by binding to the p85 regulatory subunit and there is also evidence of MAPK modulation by plakoglobin. Levels of plakoglobin are controlled by the E3 ligase Trim32.

marker for breast cancer and as a potential serum biomarker for ovarian cancer $(46,47)$. Other important proteins included CapG, a potential oncogene associated with tumour invasion and metastasis (48) and the cathepsins, which are proteinases involved in tumour invasion (49). Inflammatory stimuli transcriptionally driven by NF- $\kappa \mathrm{B}$, which is most likely derived from stromal cells, reportedly served an important role in the early development of cancer (21). In conclusion, the present study suggested that components of both the MAPK and PI3K/AKT signalling pathways may be dysregulated in the PDAC tumour microenvironment. These findings proposed an association between the overexpression of plakoglobin and the upregulation of the insulin/IGF and MAPK pathways in early resectable PDAC. The identification of plakoglobin as an important protein in PDAC may help to understand the mechanisms that mediate the initiation of tumorigenesis, with the prospect of modulating plakoglobin to prevent carcinogenesis providing a possible target for future therapeutic intervention; for example, targeting the negative regulator of plakoglobin, Trim-32, may be one approach. The potential involvement of $N-R A S$ in early PDAC has not been reported before and requires further investigation.

\section{Acknowledgements}

The authors would like to thank Dr Z Khan for assistance with some of the sample collections.

\section{Funding}

The present study was funded by The South African National Research Foundation (grant no. 91508; reference no. CSUR13091741850) and The South African Medical Research Council through a grant awarded to the Wits Common Epithelial Cancer Research Centre. MN was funded by The National Research Foundation (grant no. GUN: 105567).

\section{Availability of data and materials}

The datasets used and/or analysed during the current study are available from the corresponding author on reasonable request.

\section{Authors' contributions}

EN contributed to project concept and design, all experimental work, and data acquisition, analysis and interpretation. EN drafted the article. MN contributed to the design of the project, data analysis and interpretation, writing of the manuscript and obtained funding. MB and JD contributed in data acquisition. MS contributed to the project concept. GC contributed to the project concept and design, and data interpretation. All authors revised the work for intellectual content. All authors read and approved the final manuscript.

\section{Ethics approval and consent to participate}

The Human Research Ethics Committee of The University of Witwatersrand approved the collection of biological samples and data from patients with pancreatic adenocarcinoma (approval no. M150778) at Chris Hani Baragwanath Academic Hospital in Johannesburg. All patients gave informed voluntary consent. 


\section{Patient consent for publication}

All patients provided written consent for sample and data to be used for the current study.

\section{Competing interests}

The authors declare that they have no competing interests.

\section{References}

1. Siegel RL, Miller KD and Jemal A: Cancer statistics, 2016. CA Cancer J Clin 66: 7-30, 2016.

2. McCrea PD, Maher MT and Gottardi CJ: Nuclear signaling from cadherin adhesion complexes, Curr Top Dev Biol 112: 129-196, 2015.

3. Broussard JA, Getsios S and Green KJ: Desmosome regulation and signaling in disease. Cell Tissue Res 360: 501-512, 2015.

4. Aktary Z, Kulak S, Mackey J, Jahroudi N and Pasdar M: Plakoglobin interacts with the transcription factor p53 and regulates the expression of 14-3-3б. J Cell Sci 126: 3031-3042, 2013.

5. Chidgey M and Dawson C: Desmosomes: A role in cancer? Br J Cancer 96: 1783-1787, 2007.

6. Simcha I, Geiger B, Yehuda-Levenberg S Salomon D and Ben-Ze'ev A: Suppression of tumorigenicity by plakoglobin: An augmenting effect of N-cadherin. J Cell Biol 133: 199-209, 1996

7. Aktary Z, Alaee M and Pasdar M: Beyond cell-cell adhesion Plakoglobin and the regulation of tumorigenesis and metastasis. Oncotarget 8: 32270-32291, 2017.

8. Alaee M, Nool K and Pasdar M: Plakoglobin restores tumor suppressor activity of $\mathrm{p} 53^{\mathrm{R} 175 \mathrm{H}}$ mutant by sequestering the oncogenic potential of $\beta$-catenin. Cancer Sci 109: 1876-1888, 2018.

9. Huang L, Ji H, Yin L, Niu X, Wang Y, Liu Y, Xuan Q, Li L, Zhang H, Zhou X, et al: High expression of plakoglobin promotes metastasis in invasive micropapillary carcinoma of the breast via tumor cluster formation. J Cancer 10: 2800-2810, 2019.

10. Aktary Z, Chapman K, Lam L, Lo A, Ji C, Graham K, Cook L, Li L, Mackey JR and Pasdar M: Plakoglobin interacts with and increases the protein levels of metastasis suppressor $\mathrm{Nm} 23-\mathrm{H} 2$ and regulates the expression of Nm23-H1. Oncogene 29: 2118-2129, 2010.

11. Barker $\mathrm{N}$ and Clevers $\mathrm{H}$ : Catenins, Wnt signaling and cancer Bioessays 22: 961-965, 2000.

12. Besnier LS, Cardot P, Da Rocha B, Simon A, Loew D, Klein C, Riveau B, Lacasa M, Clair C, Rousset M and Thenet S: The cellular prion protein PrPc is a partner of the Wnt pathway in intestinal epithelial cells. Mol Biol Cell 26: 3313-3328, 2015.

13. Miravet S, Piedra J, Miró F, Itarte E, García de Herreros A and Duñach M: The transcriptional factor Tcf- 4 contains different binding sites for $\beta$-catenin and plakoglobin. J Biol Chem 277: 1884-1891, 2002

14. Winn RA, Bremnes RM, Bemis L, Franklin WA, Miller YE Cool C and Heasley LE: Gamma-Catenin expression is reduced or absent in a subset of human lung cancers and re-expression inhibits transformed cell growth. Oncogene 21: 7497-7506, 2002

15. Korc M: Role of growth factors in pancreatic cancer. Surg Oncol Clin N Am 7: 25-41, 1998.

16. Polireddy K and Chen Q: Cancer of the pancreas: Molecular pathways and current advancement in treatment. J Cancer 7: $1497-1514,2016$

17. Ji B, Tsou L, Wang H, Gaiser S, Chang DZ, Daniluk J, Bi Y, Grote T, Longnecker DS and Logsdon CD: Ras activity levels control the development of pancreatic diseases. Gastroenterology 137 1072-1082, 2009.

18. Krah NM, De La O JP, Swift GH, Hoang CQ, Willet SG, Chen Pan F, Cash GM, Bronner MP, Wright CV, MacDonald RJ and Murtaugh LC: The acinar differentiation determinant PTF1A inhibits initiation of pancreatic ductal adenocarcinoma. Elife: Jul 7, 2015 (Epub ahead of print). doi: 10.7554/eLife.07125.

19. Lee AYL, Dubois CL, Sarai K, Zarei S, Schaeffer DF, Sander M and Kopp JL: Cell of origin affects tumour development and phenotype in pancreatic ductal adenocarcinoma. Gut: Jan 23 , 2018 (Epub ahead of print). doi: 10.1136/gutjnl-2017-314426.

20. Jones S, Zhang X, Parsons DW, Lin JC, Leary RJ, Angenendt $P$, Mankoo P, Carter H, Kamiyama H, Jimeno A, et al: Core signaling pathways in human pancreatic cancers revealed by global genomic analyses. Science 321: 1801-1806, 2008.
21. Karanikas M, Esempidis A, Chasan ZT, Deftereou T, Antonopoulou M,Bozali F, Amarantidis K and Man YG: Pancreatic cancer from molecular pathways to treatment opinion. J Cancer 7: 1328-1339, 2016.

22. Long J, Liu Z, Wu X, Xu Y and Ge C: Gene expression profile analysis of pancreatic cancer based on microarray data. Mol Med Rep 13: 3913-3919, 2016.

23. McCleary-Wheeler AL, McWilliams R and Fernandez-Zapico ME: Aberrant signaling pathways in pancreatic cancer: A two compartment view. Mol Carcinog 51: 25-39, 2012.

24. Preis $M$ and Korc M: Signaling pathways in pancreatic cancer. Crit Rev Eukaryot Gene Expr 21: 115-129, 2011.

25. Mohelnikova-Duchonova B, Oliverius M, Honsova E and Soucek P: Evaluation of reference genes and normalization strategy for quantitative real-time PCR in human pancreatic carcinoma. Dis Markers 32: 203-210, 2012.

26. Bustin SA, Benes V, Garson JA, Hellemans J, Huggett J, Kubista M, Mueller R, Nolan T, Pfaffl MW, Shipley GL, et al: The MIQE guidelines: Minimum information for publication of quantitative Real-Time PCR experiments. Clin Chem 55: 611-622, 2009

27. Pfaffl MW, Horgan GW and Dempfle L: Relative expression software tool (REST) for group-wise comparison and statistical analysis of relative expression results in real-time PCR. Nucleic Acids Res 30: e36, 2002.

28. Croft D, O'Kelly G, Wu G, Haw R, Gillespie M, Matthews L, Caudy M, Garapati P, Gopinath G, Jassal B, et al: Reactome: A database of reactions, pathways and biological processes. Nucleic Acids Res 39: D691-D697, 2011.

29. Mi H, Guo N, Kejariwal A and Thomas PD: PANTHER version 6: Protein sequence and function evolution data with expanded representation of biological pathways. Nucleic Acids Res 35: D247-D252, 2007.

30. Knudsen KA and Wheelock MJ: Plakoglobin, or an 83-kD homologue distinct from beta-catenin, interacts with E-cadherin and N-cadherin. J Cell Biol 118: 671-679, 1992.

31. Rubinfeld B, Souza B, Albert I, Munemitsu S and Polakis P: The APC protein and E-cadherin form similar but independent complexes with alpha-catenin, beta-catenin, and plakoglobin. J Biol Chem 270: 5549-5555, 1995.

32. Aktary Z and Pasdar M: Plakoglobin represses SATB1 expression and decreases in vitro proliferation, migration and invasion. PLoS One 8: e78388, 2013.

33. Rozengurt E, Sinnett-Smith J and Kisfalvi K: Crosstalk between insulin/insulin-like growth factor-1 receptors and $G$ protein-coupled receptor signaling systems: A novel target for the antidiabetic drug metformin in pancreatic cancer. ClinCancer Res 16: 2505-2511, 2010.

34. Bryant KL, Mancias JD, Kimmelman AC and Der CJ: KRAS: Feeding pancreatic cancer proliferation. Trends Biochem Sci 39: 91-100, 2014

35. Posch C and Ortiz-Urda S: NRAS mutant melanoma-undrugable? Oncotarget 4: 494-495, 2013.

36. Troiani T, Martinelli E, Capasso A, Morgillo F, Orditura M, De Vita $\mathrm{F}$ and Ciardiello F: Targeting EGFR in pancreatic cancer treatment. Curr Drug Targets 13: 802-810, 2012.

37. Bruns CJ, Solorzano CC, Harbison MT, Ozawa S, Tsan R, Fan D, Abbruzzese J, Traxler P, Buchdunger E, Radinsky R and Fidler IJ: Blockade of the epidermal growth factor receptor signaling by a novel tyrosine kinase inhibitor leads to apoptosis of endothelial cells and therapy of human pancreatic carcinoma. Cancer Res 60: 2926-2935, 2000.

38. Morgan MA, Parsels LA, Kollar LE, Normolle DP, Maybaum J and Lawrence TS: The combination of epidermal growth factor receptor inhibitors with gemcitabine and radiation in pancreatic cancer. Clin Cancer Res 14: 5142-5149, 2008.

39. Pan H, Gao F, Papageorgis P, Abdolmaleky HM, Faller DV and Thiagalingam S: Aberrant activation of gamma-catenin promotes genomic instability and oncogenic effects during tumor progression. Cancer Biol Ther 6: 1638-1643, 2007.

40. Cohen S, Lee D, Zhai B, Gygi SP and Goldberg AL: Trim32 reduces $\mathrm{PI} 3 \mathrm{~K}-\mathrm{Akt}$-FoxO signaling in muscle atrophy by promoting plakoglobin-PI3K dissociation. J Cell Biol 204: 747-758, 2014.

41. Roy SK, Srivastava RK and Shankar S: Inhibition of PI3K/AKT and MAPK/ERK pathways causes activation of FOXO transcription factor, leading to cell cycle arrest and apoptosis in pancreatic cancer. J Mol Signal 5: 10, 2010.

42. Spindler V, Dehner C, Hübner S and Waschke J: Plakoglobin but not desmoplakin regulates keratinocyte cohesion via modulation of p38MAPK signaling. J Invest Dermatol 134: 1655-1664, 2014. 
43. Heidenreich KA and Kummer JL: Inhibition of p38 mitogen-activated protein kinase by insulin in cultured fetal neurons. J Biol Chem 271: 9891-9894, 1996.

44. Sweeney G, Somwar R, Ramlal T, Volchuk A, Ueyama A and Klip A: An inhibitor of p38 mitogen-activated protein kinase prevents insulin-stimulated glucose transport but not glucose transporter translocation in 3T3-L1 adipocytes and L6 myotubes. J Biol Chem 274: 10071-10078, 1999.

45. Li Y, Yu WH, Ren J, Chen W, Huang L, Kharbanda S, Loda M and Kufe D: Heregulin targets gamma-catenin to the nucleolus by a mechanism dependent on the DF3/MUC1 oncoprotein. Mol Cancer Res 1: 765-775, 2003.

46. Fabre-Lafay S, Monville F, Garrido-Urbani S, Berruyer-Pouyet C, Ginestier C, Reymond N, Finetti P, Sauvan R, Adélaïde J, Geneix $\mathrm{J}$, et al: Nectin-4 is a new histological and serological tumor associated marker for breast cancer. BMC Cancer 7: 73, 2007.
47. Derycke MS, Pambuccian SE, Gilks CB, Kalloger SE, Ghidouche A, Lopez M, Bliss RL, Geller MA, Argenta PA, Harrington KM and Skubitz AP: Nectin 4 overexpression in ovarian cancer tissues and serum: Potential role as a serum biomarker. Am J Clin Pathol 134: 835-845, 2010.

48. Zhu WY, Hunag YY, Liu XG, He JY, Chen DD, Zeng F, Zhou JH and Zhang YK: Prognostic evaluation of CapG, Gelsolin, P-gp, GSTP1, and Topo-II Proteins in non-small cell lung cancer. Anat Rec (Hoboken) 295: 208-214, 2012.

49. Gocheva V and Joyce JA: Cysteine cathepsins and the cutting edge of cancer invasion. Cell Cycle 6: 60-64, 2007. 